\title{
Improved performance of SPR sensors by a chemical etching of tapered optical fibers
}

\author{
Natalia Díaz Herrera a , Óscar Esteban ${ }^{\mathrm{b}, *}$, María-Cruz Navarrete ${ }^{\mathrm{c}}$, Agustín González-Cano ${ }^{\mathrm{a}}$, \\ Elena Benito-Peña ${ }^{\mathrm{d}}$, Guillermo Orellana ${ }^{\mathrm{d}}$ \\ a Optics Department, School of Optics, Complutense University of Madrid, Arcos de Jalón 118, 28037, Madrid, Spain \\ b Electronics Department, Alcala University, Polytechnic School, 28871 Alcalá de Henares, Madrid, Spain

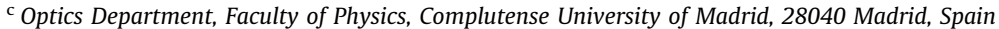 \\ d Department of Organic Chemistry, Faculty of Chemistry, Complutense University of Madrid, 28040 Madrid, Spain
}

\section{A R T I C L E I N F O}

\section{Article history:}

Received 28 February 2011

Received in revised form

21 March 2011

Accepted 23 March 2011

Available online 20 April 2011

Keywords:

Surface plasmon resonance

Tapered optical fibers

Hydrofluoric acid

Fiber-optic sensors

\begin{abstract}
A B S T R A C T
We present the results of a chemical attack on the optical fiber surface previous to the deposition of the double layer (metal plus dielectric) in Double-layer uniform-waist tapered fibers (DLUWTs) used for the development of SPR sensors. It is shown how this simple chemical treatment increases the roughness of the surface and permits improvement of the stability of the deposits and the general performance of the sensors. The obtained devices are robust and very compact, their sensitivity is good and repeatability of the measurements is remarkably increased. The procedure can be useful for any fiber-optic sensor.
\end{abstract}

(C) 2011 Elsevier Ltd. All rights reserved.

\section{Introduction}

Surface plasmon resonance is nowadays a key technology in the field of chemical and biological analysis [1]. Most of the proposed chemical and biological sensors are based on the so-called Kretchmann configuration, where the mechanism of attenuated total reflection provides the evanescent field needed to excite plasma waves on flat metallic surfaces deposited on the reflective element. Optical fibers have proved their efficiency in this context, presenting some advantages like small size, compactness and robustness. Optical fiber SPR sensors can be used for real time and in situ field measurements even in hazardous or not too accessible locations [2]. The use of tapered fibers [3-5] has proven to be very convenient for the development of high-performance transducers, especially in the form of double-layer uniform-waist fiber tapers (DLUWTs) [6-9].

However, due to the small size of these devices, the available surface to attach the ligands when a specific analyte is needed to be detected can compromise the overall sensitivity to that analyte. Although a longer taper waist can contribute to the increase of the available surface, a different approach has been considered in this work in order to keep the final length of the

\footnotetext{
* Corresponding author. Tel.: +34918856691.

E-mail address: oscar@depeca.uah.es (Ó. Esteban).
}

sensor within the limits of easy handling. We have induced roughness on the surface of the taper waist with a chemical etching of the fiber in vapor phase that gives an increase in the region of interaction, which is also interesting for fluorescence sensors based on evanescent field $[10,11]$. With this procedure, we also get a remarkable reliability and stability in the fabricated transducers that was not obtained with non-attacked fibers.

In this paper, we demonstrate how the good performance of DLUWTs can be further improved by a chemical treatment of the fiber-optic surface before the deposition of the layers, which is crucial in a field as demanding as chemical analysis, and we describe a very convenient and easy way to apply such technique for the production of SPR fiber sensors.

\section{Samples preparation}

The fabrication of tapered fibers has already been reported [7]. It is fundamentally based on the local heating of a glass fiber to its melting point while it is gently stretched in opposite directions. With this procedure it is possible to obtain the so-called adiabatic tapers with a smooth transition between the unaltered optical fiber and the waist of the taper and very low losses in the transmission, namely below $0.2 \mathrm{~dB}$. The optical fiber we have used is a single mode one at $820 \mathrm{~nm}$ nominal wavelength, and the fabricated tapers have a $35 \mu \mathrm{m}$ diameter and $15 \mathrm{~mm}$ length 
uniform waist, with a total length (including the transition regions) of $60 \mathrm{~mm}$.

The surface of optical fibers, both unaltered and tapered, presents very small or no roughness as can be seen in Fig. 1a. Thus, to induce a surface structure a chemical etching was used. Usually, the chemical etching of glass fibers has been performed in aqueous solution to obtain the fiber tapering [12-14]. By following this method, a buffered etching solution of hydrofluoric acid (BHF) was prepared and pumped by a Gilson Minipuls peristaltic pump through a PTFE column inserted in hydrofluoric acid-compatible tubing. The flow was kept at $125 \mu \mathrm{Lmin}^{-1}$ for $20 \mathrm{~min}$ up to $1 \mathrm{~h}$. The BHF reagent is an aqueous solution of hydrofluoric acid (10.5 $\mathrm{mol} \mathrm{L}^{-1}$, for trace analysis, $\geq 49 \%$, Fluka) and ammonium fluoride $\left(7.5 \mathrm{~mol} \mathrm{~L}^{-1},+99.99 \%\right.$ trace metals basis, Aldrich) at $\mathrm{pH}$ 5.5. The addition of $\mathrm{NH}_{4} \mathrm{~F}$ to $\mathrm{HF}$ controls the $\mathrm{H}^{+}$concentration and, in this way, the etching rate is kept constant. By treating the fiber with this procedure no remarkable effects are obtained in terms of roughness, and only a further decrease of the taper waist is produced. The resultant surface after etching is shown in fig. 1b.

A second procedure was tested by placing the taper in a sealed container with a $40 \%$ aqueous solution of $\mathrm{HF}$ so that the taper is above the HF level. Although the etching with HF has already been reported for the sensors and optical fiber technology field [12-14], this is the first time, to the best of our knowledge, that is being used in vapour phase to induce surface roughness. Once the taper is placed in this way, it is exposed to HF vapour at equilibrium pressure for different time intervals from 1 to 30 min while the transmittance of the device is measured online. The upper limit of this exposure time comes from the break down limit of the fiber, which is around $40 \mathrm{~min}$. The SEM (Scanning Electron Microscopy) pictures for 5 and $10 \mathrm{~min}$ of $\mathrm{HF}$ gas etching of the surface of the tapers are shown in Fig. 2a and b, respectively.
By comparing Figs. $1 \mathrm{~b}$ and 2 it is easy to see that the gas etching is the most convenient procedure to induce a structure on the surface, which in our case takes the form of craters randomly distributed of several diameters and depths. Thus, a tapered fiber etched in this way presents a larger surface available to bound ligands when a specific recognition is needed. Since the crater formation contributes to the fiber weakening, and the effect is cumulative as the exposition time increases, a trade-off must be adopted. A gas etching of $10 \mathrm{~min}$ has been used to increase the surface roughness without significant loss of mechanical strength of the taper.

After the taper etching, a double layer of $8 \mathrm{~nm}$ of aluminium and $50 \mathrm{~nm}$ of titanium dioxide has been asymmetrically deposited in order to compare their performance with previous results [7].

\section{Experimental results}

\subsection{Plasmon resonance in chemically etched DLUWTs}

The devices fabricated by the procedure explained in the above paragraph have been characterized by following the usual method [6,7], in which the spectral transmittance of the device for several values of the refractive index of the outer medium is measured. The setup for the sensing characterization is shown in Fig. 3, where a wide-spectrum light source is launched through the fiber and the spectral transmittance at the distal end is recorded with a CCD spectrometer. Since the SPR is a polarization-dependent phenomenon, a high contrast polarizer and a set of Lefévre loops have been included in the setup to obtain the maximum contrast in the transmittance minimum associated with the SP excitation, although we have already shown that the dependence of DLUWTs with polarization is not critical $[8,9]$.
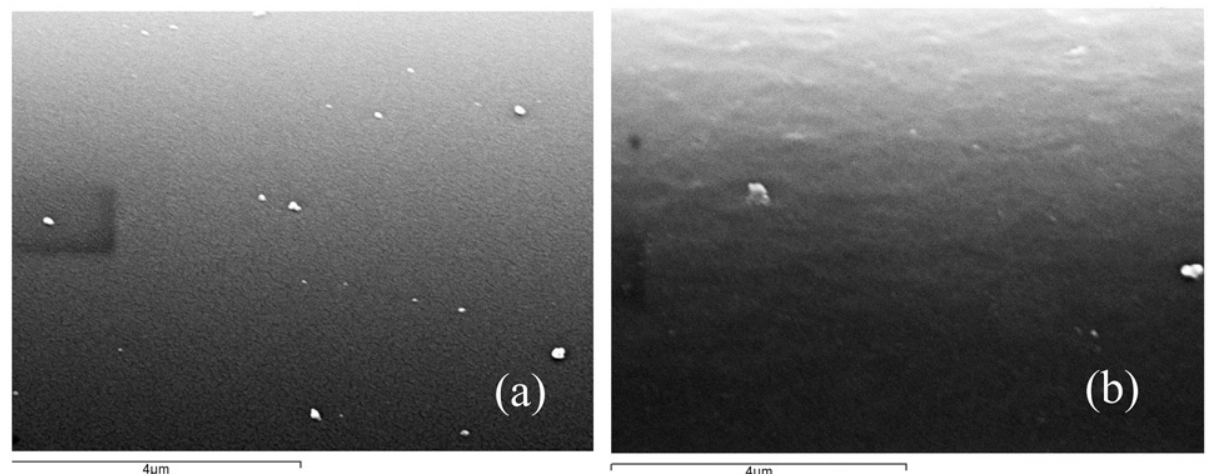

Fig. 1. (a) SEM picture of the surface of an optical fiber after tapering. (b) Surface of a tapered fiber after $1 \mathrm{~h}$ etching with the buffered HF solution.
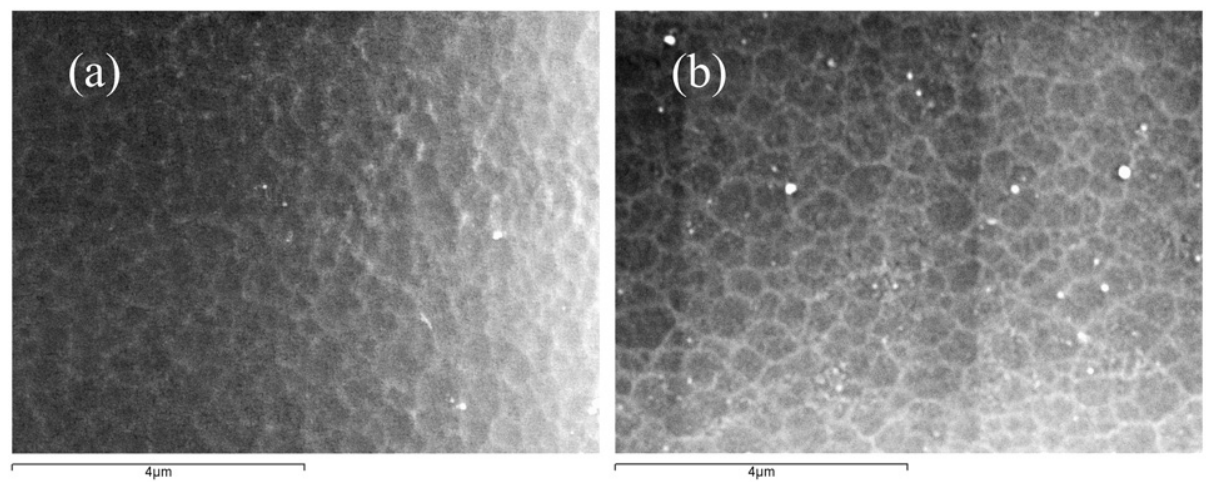

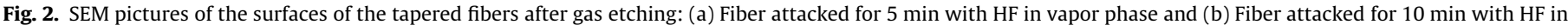
vapor phase. 


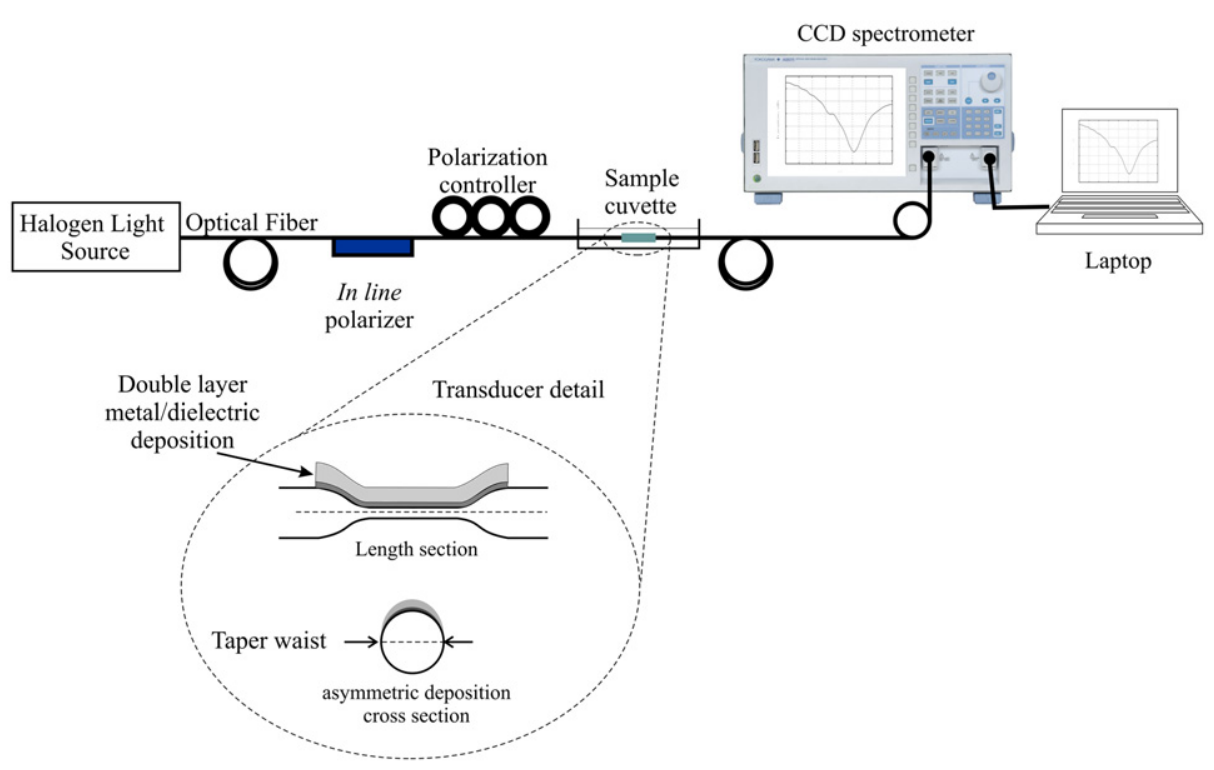

Fig. 3. Experimental setup for the characterization of the sensors.

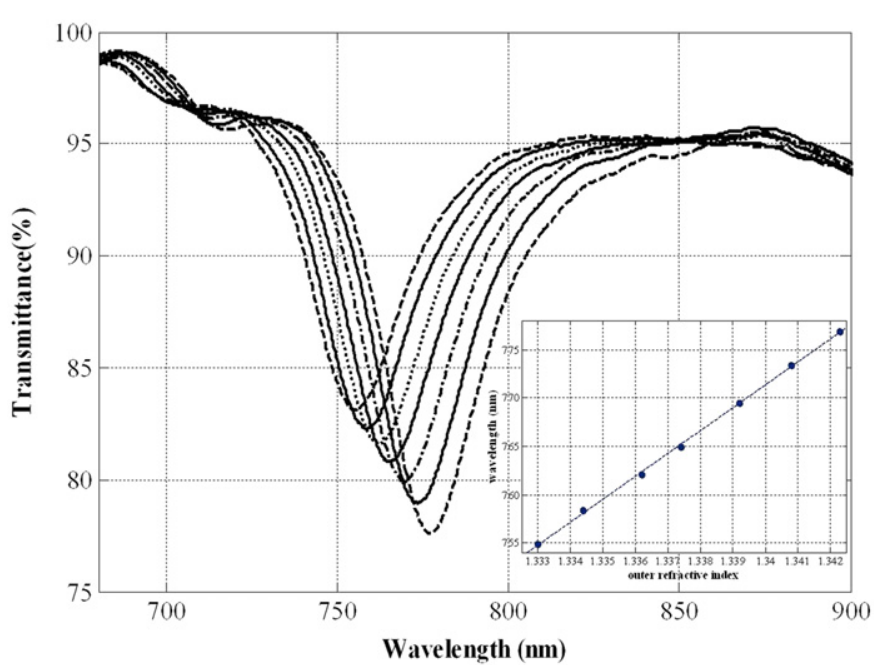

Fig. 4. Spectral transmittance curves for a DLUWT attacked 10 min with HF in vapor phase for the refractive index of the outer medium of, from left to right, $1.3330,1.3344,1.3362,1.3374,1.3392,1.3408$ and 1.3423 . As inset, the value of the transmittance minimum versus outer refractive index has been plotted. Slope of the curve gives the average sensitivity of about $2400 \mathrm{~nm} / \mathrm{RIU}$.

The transmission of the fiber when the outer medium is air is used as reference for all measurements in a refractive index range from 1.333 to 1.3423 , measured with an Abbe refractrometer, which is obtained from mixtures of pure water and ethylene glycol.

In Fig. 4 we show the typical experimental results of the characterization of one of our chemically-treated DLUWT. As it can be observed a well defined dip is present in the curve, evidencing a plasmon resonance. It is interesting to note that although the deposit was asymmetric, we have only one prominent dip and a weak secondary one. This is due to the fact that the induced roughness tends to randomize the thickness gradient due to the geometry of the deposit, and somehow the different potential plasmon resonances merge into a wider one. These results are remarkable in terms of the fundamental aspects of the phenomenology of plasmon excitation in fibers.

The appearance of plasmon resonances in the acid-treated structures shows that the chemical attack does not eliminate the possibility of exciting plasma waves in the deposited layers. In

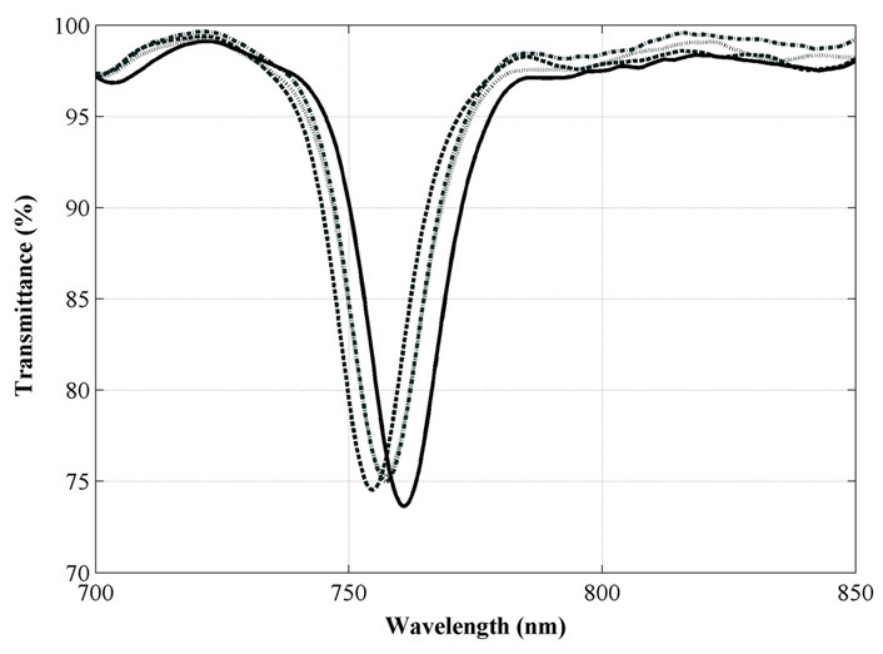

Fig. 5. Spectral transmittance of a smooth DLUWT for several successive immersions in pure water. A shift in the transmittance minimum is clearly observed for each one.

fact the behavior of the device is good in terms of sensitivity since, from the slope of the inset in Fig. 4, where the wavelength of the transmittance minimum for several refractive index values is plotted, we can estimate an overall sensitivity of around $2400 \mathrm{~nm} / \mathrm{RIU}$, which is a figure comparable to that typically obtained with the traditional transducers (ca. $3000 \mathrm{~nm} / \mathrm{RIU}$ ) [6-9]. Therefore, the performance of the DLUWTs after the treatment is good enough to be used as refractive index sensors.

3.2. Stability of the deposits and repeatability of the measurements in successive immersions

The chemical etching of the fiber surfaces and the subsequent roughness they acquire have also a very interesting side-effect, namely an improvement in the stability and durability of the deposits and a remarkable increase in the repeatability of the measurements. Indeed, when a double layer is deposited on a non-flat smooth surface, the behaviour of the transducer very often shows some drift in a series of successive immersions in water, as shown in Fig. 5, where the position of the plasmon 


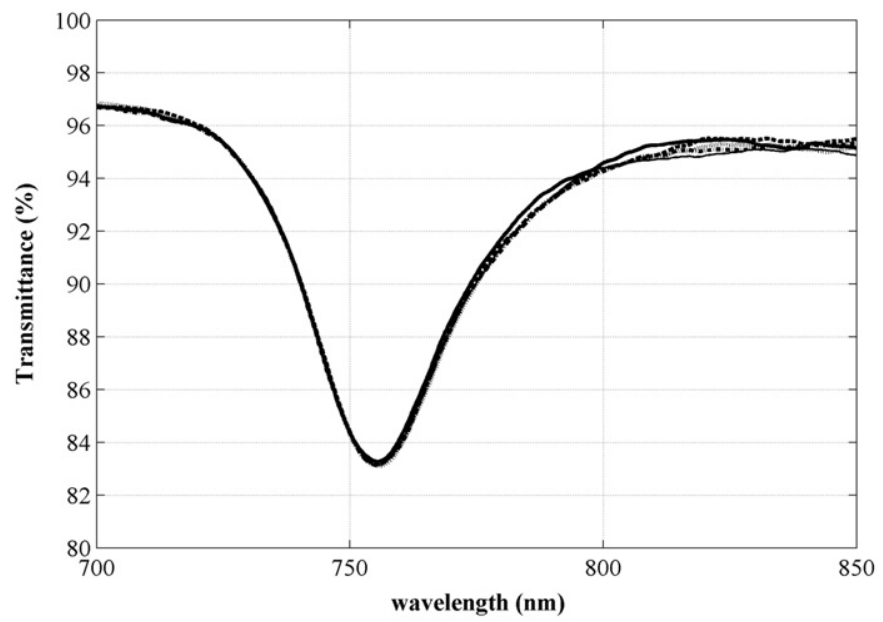

Fig. 6. Spectral transmittance curves for a DLUWT attacked $10 \mathrm{~min}$ with HF in vapor phase. Various curves correspond to successive immersions in water. No drift in the position of the minimum is observed.

$\operatorname{dip}(\mathrm{s})$ moves to higher wavelengths as the DLUWTs are immersed for a sequence of measurements. Although this effect does not compromise the overall behavior of a sensor in terms of sensitivity, it certainly makes it mandatory to establish a calibration point before performing the measurement. Such necessity can be an undesirable procedure when a long distance in-situ measurement is to be made, and can affect the dynamic range and the repeatability of the measurements.

As we can see in Fig. 6, the effect of the chemical treatment in this particular case is highly beneficial. We see the results of up to 15 successive immersions with no significant drift of the plasmon dip. In this context, it is quite possible that the increased roughness of the fiber surfaces provides better adherence for the layers. Thus, the simple chemical treatment proposed seems to be ideal as a pre-processing step in the deposition of any layer in curved fiber geometries and provides greatly improved repeatability to the measurements.

\section{Conclusions}

A detailed study of the effect of chemically etching of the surfaces of tapered optical fibers with HF in the gas phase has been performed. It shows that a treatment of this kind is adequate in general for the improvement of the reliability of the fiber sensors developed with this kind of elements. In particular it has been shown how this procedure does not compromise the transmission characteristics of the fiber, allows plasmon resonances and notably increase the stability of the transducers and the long term repeatability of the measurements when the sensors undergo repeated immersion in water. The procedure is simple and can be recommended for optical fibers used in the field of chemical and biological sensing.

\section{Acknowledgments}

This work has been partially supported by Spanish Ministry of Science research projects SPRINT (CTQ2009-10550) and MAXMART (CTQ2009-14565-C03-01), and the Community of Madrid research grants FACTOTEM II (S2009/ESP-1781) and FUTURSEN (CM-S-505/AMB/0374) as well as the European Social Fund and the European Fund for Regional Development.

\section{References}

[1] Homola J. Surface plasmon resonance sensors for detection of chemical and biological species. Chemical Reviews 2008;108:462-493.

[2] Sharma AK, Jha R, Gupta BD. Fiber-optic sensors based on surface plasmon resonance: a comprehensive review. IEEE Sensors Journal 2008;17:1118-128.

[3] Villatoro J, Monzón-Hernández D, Mejía E. Fabrication and modelling of uniform-waist single-mode tapered optical fiber sensors. Applied Optics 2003:42:2278-83.

[4] Obando LA, Gentleman DJ, Holloway JR, Booksh KS. Manufacture of robust surface plasmon resonance fiber optic based dip-probes. Sensors and Actuators B 2004;100:439-49.

[5] Díez A, Andrés MV, Cruz JL. In-line fiber-optic sensors based on the excitation of surface plasma modes in circular metal-coated tapered fibers. Sensors and Actuators B 2001;73:95-9.

[6] Bueno FJ, Esteban Ó, Díaz-Herrera N, Navarrete MC, González-Cano A. Sensing properties of asymmetric double-layer covered tapered fibres. Applied Optics 2004;43:1615-20.

[7] González-Cano A, Bueno FJ, Esteban Ó, Díaz-Herrera N, Navarrete MC Multiple surface-plasmon resonance in uniform-waist tapered optical fibers with an asymmetric double-layer deposition. Applied Optics 2005;44: 519-26.

[8] Esteban Ó, Díaz-Herrera N, Navarrete MC, González-Cano A. SPR sensors based on uniform-waist tapered fibers in reflective configuration. Applied Optics 2006;45:7294-8.

[9] Navarrete MC, Díaz-Herrera N, González-Cano A, Esteban Ó. A polarizationindependent SPR fiber sensor. Plasmonics 2010;5:7-12.

[10] Stranik O, McEvoy HM, McDonagh C, MacCraith BD. Plasmonic enhancement of fluorescence for sensor applications. Sensors and Actuators B 2005;107: 148-153.

[11] Taitt CR, Anderson GP, Ligler FS. Evanescent wave fluorescence biosensors. Biosensors and Bioelectronics 2005;20:2470-87.

[12] Iadicicco A, Cusano A, Cutolo A, Bernini R, Giordano M. Thinned fiber bragg gratings as high sensitivity refractive index sensor. IEEE Photonics Technology Letters 2004;16:1149-51.

[13] Cibula E, Donlagic D. Low-loos semi-reflective in-fiber mirrors. Optics Express 2010;18:12017-26.

[14] Zhang EJ, Sacher WD, Poon KKS. Hydrofluoric acid flow etching of low-loss subwavelength-diameter biconical tapers. Optics Express 2010;18. 22593-98. 